\title{
Sensitivity Analysis of Estimated Weights under Incomplete Information
}

Author(s): Masanori OZAWA and Yutaka KATO

In this paper, we discuss the sensitivity analysis for performance of estimation methods under incomplete information in a comparison matrix. Since there are some no-information elements in the comparison matrix, estimated weights lack some reliability because of incomplete information. Harker's method and the least square method are known to be good methods to estimate weights under incomplete information in general. We show some properties of the estimated weights when no-information elements in a comparison matrix has a specific structure. 